\title{
A Case Study on Comparison of Male and Female Vowel Formants by Native Speakers of Gujarati
}

\section{Pooja Pravinkumar Ahuja*}

Institute of Forensic science, Gujarat Forensic Sciences University, Gujarat, India

*Corresponding author: Pooja Pravinkumar Ahuja, Assistant Professor, Institute of Forensic science, Gujarat Forensic Sciences University, Sector 18/A, Near Police Bhavan, Gandhinagar, Gujarat, India, Tel: 9724304885; E-mail: pahuja159@gmail.com

Rec date: Nov 18, 2014 Acc date: October 15, 2015 Pub date: October 19, 2015

Copyright: (C) 2015 Ahuja PP. This is an open-access article distributed under the terms of the Creative Commons Attribution License, which permits unrestricted use, distribution, and reproduction in any medium, provided the original author and source are credited.

\begin{abstract}
Compared to the amount of research on Gujarati consonants, few studies indeed have been done to analyse Gujarati vowels. In addition, the results of recent studies are mixed. This study was conducted for reviewing the Gujarati vowel system. In this study, we measured the formant frequencies of eight Gujarati vowels by three dialect female speakers of native Gujarati's in their early 30s, and compared the results with those of two Gujarati males in their early 30s. We confirmed overlapped /i, î/, /u, ù/ and /a, ā/ formant frequencies uttered by female speakers, this was also the result of male speakers. These findings were similar to the results in the previous studies. Additionally vowel spaces of males have corresponded to the results indicated in previous studies. However the vowel spaces of females were different from the results of previous studies.
\end{abstract}

Keywords: Gujarati; Vowel system; Formant measurement; Dialect

\section{Introduction}

The Gujarati vowel system is discussed by phoneticians in Gujarat state. However, studies of Gujarati vowel system have not received much attention; compared to the amount of research done on consonants, few studies have been done to analyse vowels. Therefore even the total number of vowels is still being discussed. One reason is that The National Institute of the Gujarati Language seven vowels a, ā, $\mathrm{i}, \hat{\mathrm{i}}, \mathrm{u}, \mathrm{u}, \mathrm{r}$ were confirmed as monosyllabic, and both /eh/ and /aع/ were admitted as disyllabic too [1]. The total number of monosyllabic differs according to each phonetician [2]. Also in Cho [3], though there are some prescribed monosyllabic in Gujarati, the total number of monosyllabic varies from seven to ten according to each phonetician. In fact, each phonetician has a different claim. Production experiments by Cho [3] on ten vowels were published for Gujarati Language dialect speakers. The results revealed that two vowels are disyllabic and seven vowels are monosyllabic. The most famous study result is undisposed /e/ and /ae/ in Gujarati monosyllabic. In studies, Gujarati dialect speakers had no way of distinguishing /e/ and /ae/ in a perception experiment; Cho [1] and Moon [4] also obtained the same result by production experiment for their research carried out for sui langauage. Thus, the study of Gujarati monosyllabic had focused on /e/ and $/ \mathrm{ae} /$ in the past. In recent studies, vowel spaces are paid attention to, including other monosyllabic [1-8], but each side reveals a different result. In the study by Cho [1], the tongue position in vowels $/ a /$ is more front than /a/ by males. In a study of Moon [4], /u/ and / $\overline{\mathrm{u}} /$ overlapped each other. However, the common results are just /e/ and / ae/; results for other vowels are not overlapping results. Igeta, et al. [3] revealed subsequently overlapped $/ \mathrm{u} /$ and $/ \overline{\mathrm{u}} /$ by males, as is the case with Moon [4]. As we mentioned before, these results did not correspond except for /e/ and /ae/.Thus, we conducted measuring formant frequencies (F1, F2) for a few Gujarati dialect speakers. Although we needed many participants for accurate data, we accorded priority to the conditions of participants (age, sex) rather than the quantitative number. The purpose of this study is a comparison of values of formant frequencies (F1, F2) between male [3] and female as a case study.

\section{Research Method}

The utterances of nine Gujarati vowels by native speakers of Gujarati dialect were recorded.

\section{Participants}

Three female native speakers of Gujarati dialect (KFS1, KFS2, KFS3) took part in this study. They have lived in Gujarat from birth to 15 years of age, and they were 31 years old at that time. They were all graduate students and they were able to converse fluently with native speakers of Gujarat.

\section{Recorded sounds}

Among the 110 nonsense words recorded by native speakers of Gujarati dialect, seven were monosyllabic /a, à, i, î, u, ù, r, , and 80 were combinations of ten Gujarati consonants /b, d, g, p, t, k, s/ (excluding nasal consonants and tense consonants). Twenty-two additional /V/ and /CV/ including / $\mathrm{u}, \overline{\mathrm{u}} /$ were also recorded. These 110 nonsense words were repeated three times by each participant; a total of 330 utterances were recorded. However our targets were only /V/ (/a, ā, i, î, $\mathrm{u}, \overline{\mathrm{u}}, \mathrm{r} /$ /) in this study.

\section{Procedure}

First, the nonsense words were presented one by one on the computer screen, and the utterances were recorded. We manipulated the computer manually when we showed the next nonsense words on the computer screen to the participants.

The reasons were to avoid any immixing of other noise by the participants' movement and to enable the participants to concentrate on their utterances. The recordings took place in a sound-proof room 
Page 2 of 4

(background noise level: 23.3dBA), using a digital sound recorder (Marantz PMD 660) and a microphone (SONY ECM-23F5) at a sampling frequency of $48 \mathrm{kHz}$ and a quantization level of 16 bit.

\section{Analysis}

Our targets were only V (/a, ā, i, î, u, ù, r, /); we measured the vowel formant frequencies by using XKL [5]. Some preparation was necessary before using XKL. First, these utterances were down sampled from $48 \mathrm{kHz}$ to $8 \mathrm{kHz}$, and the file format was converted from wav to XKL on Linux. Then, we measured three points of first and second formant frequencies (F1, F2) in the steady state of each vowel. We adopted estimated values of formant frequency by linear prediction analysis in XKL.

Additionally, we confirmed it by visual checks. We calculated the distance between origin $(0,0)$ and each point $(\mathrm{F} 1, \mathrm{~F} 2)$ on a plane surface of F1-F2 based on the obtained value of F1 and F2 for each of the three points in each vowel. The F1 and F2 pairs that give the median values in the three distances were adopted as the measures of central tendency in each vowel. Finally, we calculated the twodimensional normal distribution on F1 and F2 values on each vowel, and we plotted iso-probabillity ellipses in such a way that $\mu \pm 3 \sigma$ ( $\mu$ : average, $\sigma$ : standard deviation).

\section{Results and Discussion}

\section{Value of F1 and F2}

Table 1 presents average F1 and F2 by Gujarati dialect male and female speakers in their 30s. Some data of F1 and F2 of males were taken from Igeta [5].

\begin{tabular}{|c|c|c|c|c|}
\hline \multirow[t]{2}{*}{ Target } & \multicolumn{2}{|c|}{$\mathrm{F} 1[\mathrm{~Hz}]$} & \multicolumn{2}{|c|}{ F2 [Hz] } \\
\hline & Male & Female & Male & Female \\
\hline la/ & 803 & 887 & 1175 & 1358 \\
\hline /ā/ & 217 & 238 & 2318 & 2980 \\
\hline /i/ & 284 & 468 & 1549 & 1787 \\
\hline /î/ & 268 & 356 & 876 & 1065 \\
\hline$/ \mathrm{u} /$ & 482 & 592 & 2064 & 2518 \\
\hline$/ \bar{u} /$ & 484 & 663 & 2067 & 2517 \\
\hline$|r|$ & 590 & 707 & 857 & 1138 \\
\hline lae/ & 373 & 444 & 666 & 743 \\
\hline
\end{tabular}

Table 1: Average F1 and F2 by Gujarati dialect male and female speakers in their early 30 s.

\section{Individual data}

First, we discussed individual data; after that, we presented results of the plotted vowel space for female speakers. Especially we focused on three vowel pairs: /i, î/, /u, ù / and /a, ā/ [6-8].

\section{In the case of KFS1}

Figure 1 indicates the vowel space of KFS1. In the case of KFS1, /u, $\overline{\mathrm{u}} /$ overlapped completely with [3-5], and /a, à/ overlapped, too. The isoprobability ellipse of /a, $\bar{a} /$ overlapped on F2. The reason is the difference of front-back of tongue. This result corresponded to [5]; however, /i, il/ did not overlap, so this result did not correspond to $[3,4]$.

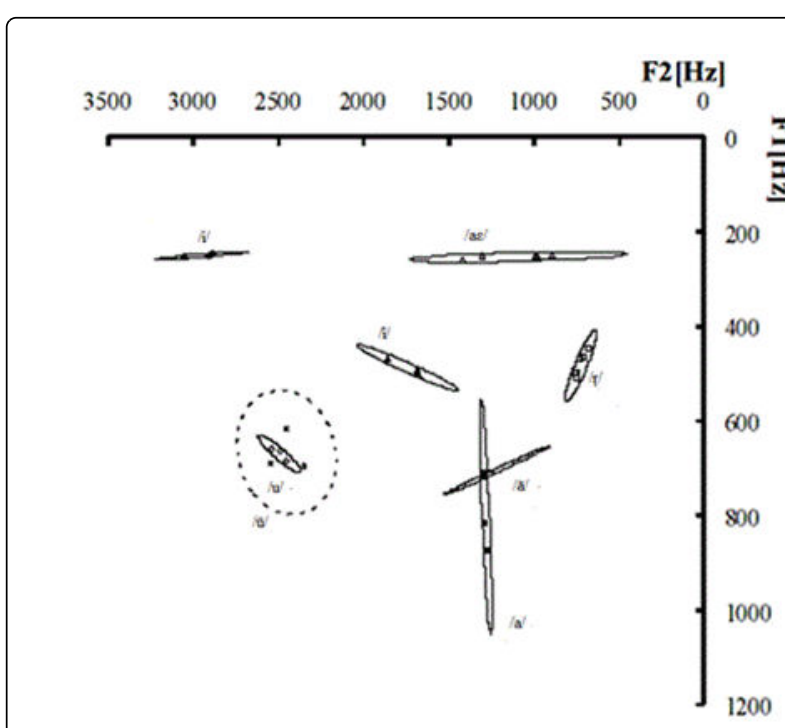

Figure 1: Vowel space of KFS1.

\section{In the case of KFS2}

Figure 2 shows the vowel space of KFS2. In the case of KFS2, the /i, $\hat{1} /$ overlapped; thus the result is identical with [3-5], but the shape of the isoprobability ellipse of $/ \mathrm{i} /$ is very long and thin. The reason is that the difference of height of tongue put in an appearance at value of $\mathrm{F} 1$ $[9,10]$.

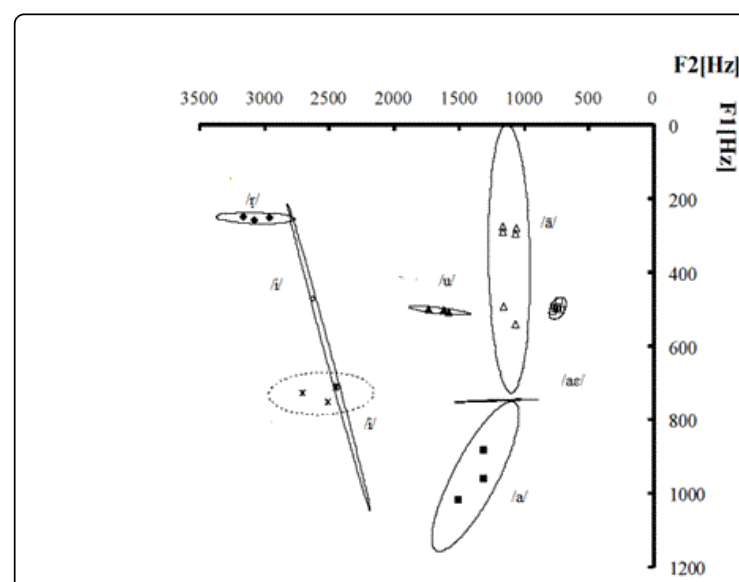

Figure 2: Vowel space of KFS2.

\section{In the case of KFS3}

Figure 3 shows the result of the vowel space of KFS3. In the case of $\mathrm{KFS} 3, / \mathrm{i}, \hat{\mathrm{i}} /$ overlapped the shape of the iso-probability ellipse of / $\hat{\mathrm{i}} / \mathrm{is}$ very thin, because the shape was related to the front-back position of the tongue. However, /u, ù/, /a, à/ did not overlap. 
Page 3 of 4

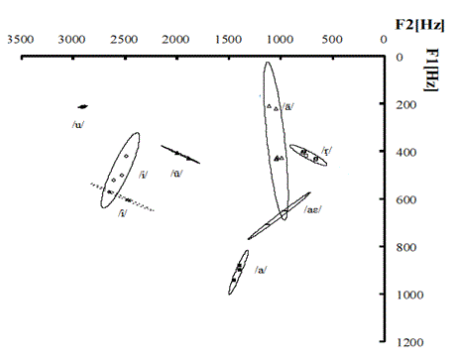

Figure 3: Vowel space of KFS3.

\section{Comparing female and male}

The results are shown in Figures 4 and 5. Figure 4 illustrates the vowel space of Gujarati dialect female speakers in their 30s, and Figure 5 is males' (Igeta [5]) data [11].

In Figure 4, /i, î/ nearly overlapped. The /a, r, overlapped on F1, and /a, à/ overlapped on F2. The iso-probability ellipse of /i, î/ indicates a difference of the height of tongue; that of $/ \mathrm{a}, \overline{\mathrm{a}} /$ is related to the frontback position of the tongue. ICPhS XVII Regular Session Hong Kong, 17-21 August 2011937

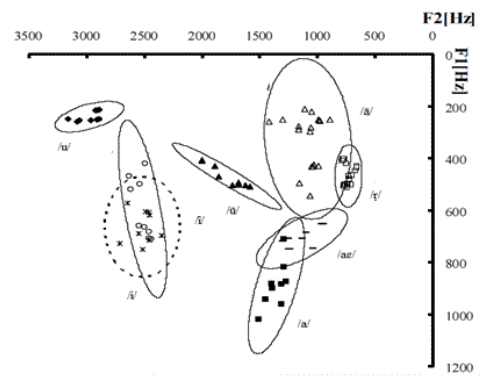

Figure 4: The illustration of the vowel space of Gujarati dialect female speakers in their $30 \mathrm{~s}$

In Figure $5, / \mathrm{i} /$ and $/ \hat{\mathbf{i}} /$ overlapped. The result in the present study is consistent with the finding of $[3,4]$, and $/ \mathrm{u}, \overline{\mathrm{u}} /, / \mathrm{a}, \overline{\mathrm{a}} /$ overlapped. The result of $/ \mathrm{u}, \overline{\mathrm{u}} /$ and $/ \mathrm{a}, \overline{\mathrm{a}} /$ corresponded with $[3,4]$. We confirmed overlapping of the same vowel pairs: /i, î/, /u, $\overline{\mathrm{u}} /, / \mathrm{a}, \overline{\mathrm{a}} /$ comparing Figures 4 and 5 [12].

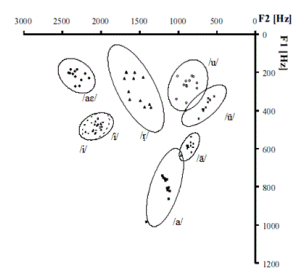

Figure 5: The illustration of the vowel space of Gujarati dialect male speakers in their $30 \mathrm{~s}$.

\section{Summary of results}

We organized overlapped vowel spaces based on review studies [3-5]. Table 2 gives a summary. This study revealed that vowel space of males have corresponded with the results indicated in the previous studies. However those of females were different from the results of previous studies.

Table 2 shows the individual differences that appeared in the result; these covered the results of the previous study totally.

\begin{tabular}{|l|l|l|l|l|}
\hline & li, îl & lu, ūl & la, āl & Other overlaps \\
\hline KFS1 & Overlapped & & Overlapped & \\
\hline KFS2 & Overlapped & & & $/ \mathrm{i}, \mathrm{r} /$ \\
\hline KFS3 & Overlapped & & & $/ \mathrm{a}, \mathrm{a} /$ \\
\hline Female & Overlapped & Overlapped & Overlapped & / a, $\mathrm{r} /$ \\
\hline Male & Overlapped & Overlapped & Overlapped & \\
\hline
\end{tabular}

Table 2: Overlapped monosyllabic based on the results of individuals.

\section{Conclusion}

There were basically two results on vowel space. The vowel space of female is larger than male space; however, individual data did not perfectly correspond to the previous studies. The result of overlapped /a, â/ corresponded to Igeta [5]; this result was revealed for the first time there. The results in the present study were consistent with the finding of Igeta [5]. Because of a potential of other overlapped cases for when there were differences between individuals, we need to research native speakers of Gujarati dialect living in Gujarat in the near future. Furthermore, the three vowel pairs overlapped on the isoprobabillity ellipse; however, we have to investigate the overlapping data.

\section{Acknowledgments}

This study work was supported by the Directorate of Forensic Science, Gandhinagar, Gujarat, India.

\section{References}

1. Lee IS, Lee SO, Choi W (2004) Korean Language.

2. Noma H (2007) Kankokugo Kyooikuron Kouza. (In Japanese), Kuroshio Publisher.

3. Cho SM (2003) An acoustic study of Korean vowel system. (In Korean). Korean Language and Culture 24: 427-441.

4. Moon S (2005) A fundamental phonetic investigation of seoul monosyllabi. (In korea). Malsori 62: 1-17.

5. Igeta T, Arai T (2011) Formant measurement of vowels spoken by Korean Seoul dialect speakers: A case study for reviewing vowel system. (In Japanese. Proc 2011 Spring Meeting Acoustical Society of Japan 463-466.

6. Chung HY, Makino S, Kido K (1988) J Acoust Soc Jpn 9: 225-232.

7. Kasuya H, Suzuki H, Kido K (1968) Change in pitch and first three formant frequencies of five Japanese vowels with age and sex of speakers. (In Japanese) Acoust Soc Japan 24: 355-364.

8. Klatt DH (1984) Speech Communication Group Working Papers IV. Cambridge: MIT, Research Laboratory of Electronics 73-82.

9. Magen HS, Blumstein SE (1993) Effect of speaking rate on the vowel length distinction in Korean. J Phonetics 21: 387-409. 
Citation: Ahuja PP (2015) A Case Study on Comparison of Male and Female Vowel Formants by Native Speakers of Gujarati. J Forensic Res S4: S4-004. doi:10.4172/2157-7145.1000S4-004

Page 4 of 4

10. Peterson GE, Barney HL (1952) Control methods used in a study of the vowels. J Acoust Soc Am 24: 175-184.

11. Umeda H (1995) Age differentiation of the vowel system in the Seoul Korean: Acoustic measurements. J Asian and African Stud 48-49.
12. Yang B (1992) An acoustical study of Korean monosyllabic produced by male and female speakers. J Acoust Soc Am 91: 2280-2283.

This article was originally published in a special issue, entitled: "Forensic Science \& Technology", Edited by Amarnath Mishra, Associate Professor, Department of Forensic Medicine, Tribhuvan University 